\title{
L'Hellénisme pontique et sa diaspora : les territoires de la mémoire
}

Singularité géographique du Pont et continuité de l'Hellénisme

Pontic Hellenism and its Diaspora: Territories of Memory

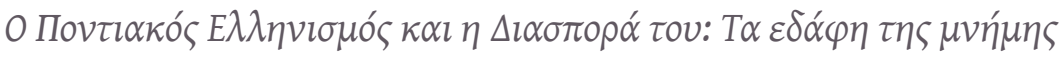

\section{Michel Bruneau}

\section{CpenEdition}

Journals

Édition électronique

URL : https://journals.openedition.org/ceb/17662

DOI : $10.4000 /$ ceb. 17662

ISSN : 2261-4184

Éditeur

INALCO

Édition imprimée

ISBN : 9782858313693

ISSN : 0290-7402

Référence électronique

Michel Bruneau, «L'Hellénisme pontique et sa diaspora : les territoires de la mémoire », Cahiers balkaniques [En ligne], 47 | 2020, mis en ligne le 21 août 2020, consulté le 06 juillet 2021. URL : http:// journals.openedition.org/ceb/17662 ; DOI : https://doi.org/10.4000/ceb.17662

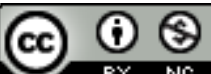

Cahiers balkaniques est mis à disposition selon les termes de la Licence Creative Commons Attribution - Pas d'Utilisation Commerciale 4.0 International. 


\title{
L'Hellénisme pontique et sa diaspora : les territoires de la mémoire Singularité géographique du Pont et continuité de l'Hellénisme
}

\author{
Pontic Hellenism and its Diaspora: Territories of Memory

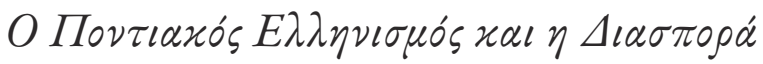

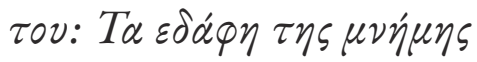

Michel Bruneau CNRS-Bordeaux

Les Grecs pontiques et leurs descendants sont environ un million en Grèce. Tous les chrétiens orthodoxes ont dû quitter leurs territoires et lieux d'origine du Pont et plus généralement d'Anatolie en 1923-1924 à la suite du traité de Lausanne (1923). En Grèce, et ailleurs dans le monde, ils revendiquent leur identité à travers une vie associative très active et cultivent une mémoire qui a toujours un lien à leurs lieux d'origine. De tous les Grecs originaires de l'espace de l'actuel État-nation turc, ils sont de loin ceux qui affirment le plus vigoureusement leur identité. Leur attachement extrême à leur terre peut être constaté tout au long de leur histoire. Leur nostalgie ne s'éteint jamais et, du Pont, fut transférée en Grèce.

Pourquoi ne se sont-ils pas totalement assimilés en Grèce et dans leurs autres pays d'accueil ? Pourquoi, de la deuxième à la quatrième et bientôt la cinquième génération, continuent-ils de se référer à leur identité et à leur « patrie » du Pont-Euxin, du rivage de la mer Noire et des montagnes qui la bordent ? Pour comprendre ce phénomène géoculturel et géopolitique, il faut analyser la façon dont ils ont construit un espace migratoire transnational entre Pont, Caucase, Grèce, et au sein de la diaspora grecque du Nouveau Monde et d'Europe occidentale, comment ils se sont ponctuellement ré-enracinés sur les lieux de leur accueil. Il faut voir aussi comment ils ont su se constituer une « iconographie » 
particulièrement riche et utiliser, entretenir leur culture religieuse et profane pour continuer à exister en tant que peuple distinct au sein de l'Hellénisme. Comment ce peuple, qui a perdu de manière quasiment irréversible son territoire d'origine, sa « patrie », en conserve-t-il la mémoire et construit-il son identité en diaspora en créant des lieux, voire des hauts lieux, porteurs d'une iconographie ? Dans quelle mesure lui permettent-ils de transmettre cette identité territoriale d'une génération à l'autre ?

L'attachement passionné des Grecs pontiques à leur patrie d'origine, leur identité et leur culture grecques mieux protégées dans le Pont que dans le reste de l'Anatolie, n'étaient-ils pas liés à la singularité et à l'isolement de ce territoire par rapport aux autres territoires de l'Hellénisme en Asie Mineure ?

Le Pont correspond au territoire de l'ancien Empire de Trébizonde (1204-1461) des Grands Comnènes, fondé après la prise et le saccage de Constantinople le 12 avril 1204 par les Latins, en particulier Vénitiens, de la quatrième croisade. Il constitue une unité géographique close et isolée qui n'a jamais facilité l'entrée massive de peuples étrangers ou des incursions ennemies. Il comprend deux régions : la plaine côtière d'une part qui n'excède pas $50 \mathrm{~km}$ de large, mais est souvent très étroite (quelques centaines de mètres au pied de versants abrupts) ; l'intérieur montagneux d'autre part, fait de hautes chaînes et d'une succession de vallées nord-sud, parallèles d'ouest en est. Lorsque la chaîne des Alpes pontiques s'élève progressivement jusqu'à près de 4000 mètres à l'est d'Ordu, on se trouve en présence d'une « île dans la terre » selon l'expression de M. E. Meeker ${ }^{1}$ qui place cette région à part dans l'ensemble de l'Asie Mineure.

Pendant longtemps, elle a vécu hors de l'orbite des émirats turco-islamiques de l'intérieur et après la conquête et l'incorporation tardive à l'Empire ottoman (1461), elle est restée très largement non turcophone et non musulmane. Elle n'est devenue majoritairement musulmane qu'au XVII ${ }^{\mathrm{e}}$ siècle. On y trouve donc

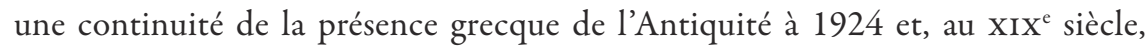
l'hellénisme y a connu une renaissance économique, culturelle et politique ${ }^{2}$. Sous la domination ottomane (Tourkokratia), la particularité géographique du Pont, en l'isolant, a aidé les Grecs à survivre et même à se développer. La zone montagneuse avec ses mines a servi de refuge, l'émigration vers les côtes du Nord et de l'Est du Pont-Euxin, en Russie, a été une échappatoire en cas d'oppression insupportable ${ }^{3}$.

1. Meeker, 2002.

2. BRYER, 1976.

3. LAMPSIDIS, 2009. 


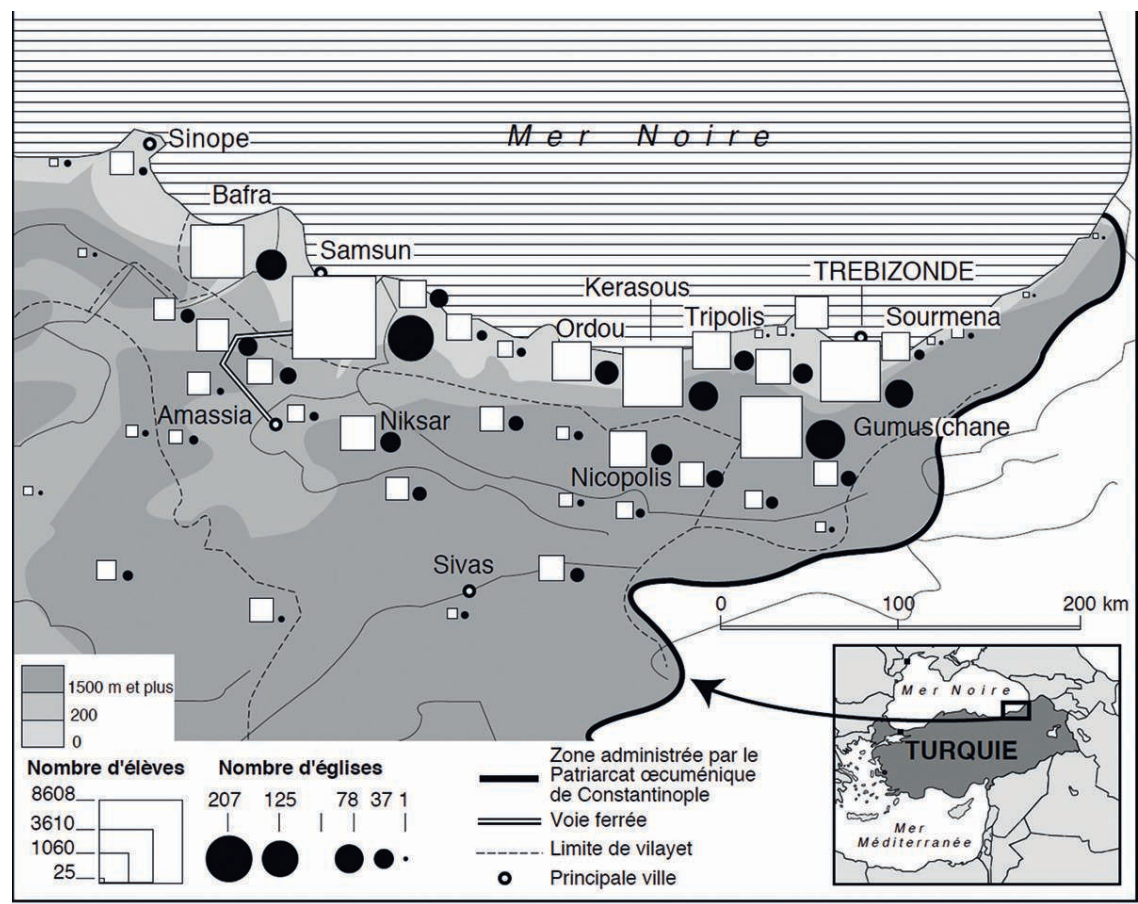

source: carte dressée par Notis Botsaris, Athènes février 1919

FIGURE 1. LES GRECS DANS LE PONT AVANT LA PREMIÈRE GUERRE MONDIALE : ÉCOLES ET ÉGLISES GRECQUES DANS LE PONT (1912)

Bruneau, 2015, p. 160.

Le morcellement en petites unités régionales, voire en villages, centres de formations sociales de type lignager ou clanique, a favorisé les patriotismes locaux qu'on retrouve au sein de la vie associative de la diaspora. Un Grec chrétien du Pont était d'abord de Sanda, Sourmena, Trapezous, Kromni, Phytiana... Il était aussi Romios, c'est-à-dire membre du millet orthodoxe dirigé par le Patriarche œcuménique de Constantinople, sujet du sultan ottoman. À la fin du XIX et au début du $\mathrm{Xx}^{\mathrm{e}}$ siècle, il se disait Ellinas, « Grec », depuis le développement d'un grand nombre d'écoles avec des instituteurs formés au Frontistirio de Trapezountas, d'Argyroupolis ou à Athènes. Ce n'est qu'après 1923 qu'il est appelé Pontios, Grec pontique, en Grèce. Son identité a donc été définie par rapport au territoire d'origine, le Pontos. Elle est devenue une identité ethnorégionale en Grèce, conjuguée à celle de réfugié, issu de l'échange des populations du traité de Lausanne (1923). 
CAHIERS BALKANIQUES

La presse allophone dans les Balkans

\section{L'espace-réseau transnational et diasporique des Grecs pontiques}

L'espace migratoire transnational des Grecs pontiques s'est constitué en quatre grandes phases chronologiques et géographiques. Dans un premier temps de longue durée, $\mathrm{du} \mathrm{Xv}^{\mathrm{e}}$ au début du $\mathrm{Xx}^{\mathrm{e}}$ siècle, ils ont émigré sur les côtes de la mer Noire et dans le Caucase russe. À partir de la Première Guerre mondiale et à la suite du traité de Lausanne (1923), leur espace migratoire s'est étendu à la Grèce et à l'Asie centrale soviétique (déportations de la période stalinienne, 1937-1950). Dans un troisième temps, après la Seconde Guerre mondiale, ils se sont redéployés dans les pays du Nouveau Monde (États-Unis, Canada, Australie) et en Europe occidentale (Allemagne, Belgique, Suède), au sein de la diaspora grecque, dont ils constituent une partie qui garde sa spécificité. Enfin, depuis la dissolution de l'URSS, environ 200000 Pontiques de Russie, d'Ukraine, d'Arménie et de Géorgie sont venus s'installer en Grèce, le plus souvent dans les villages et villes d'établissement des réfugiés de Lausanne (1923) avec lesquels ils avaient souvent des liens de parenté.

Cet espace transnational et diasporique se structure autour du Pont et de la mer Noire selon trois auréoles correspondant aux quatre phases de ces migrations mondiales : le Caucase (1829-1918), la Grèce et la Russie (1920-1950), les pays du Nouveau Monde et l'Europe occidentale (1950-1970), le « retour » en Grèce depuis les pays de l'ex-URSS (1989-2005). Étudiant les processus d'installation et de ré-enracinement dans chacun de ces types de territoires, on se limitera à un exemple choisi pour sa représentativité dans chacun d'entre eux. On analysera comment, à chacune de ces phases et dans chacun de ces territoires d'installation ou d'accueil, les Grecs pontiques, originaires des petites régions montagneuses de Sanda, d'Imera et de Kromni ont créé de nouveaux lieux en se référant à leurs territoires d'origine dans le Pont, Tsalka et Tetritskaro, Kars dans le Caucase, Nea Sanda, Nea Imera, Kromniko, Kalamaria en Grèce.

\section{Les migrations vers le Caucase et la construction de lieux et de territoires rappelant les «patries $\gg$ de l'origine}

Les migrants originaires des petites régions montagneuses du Pont se sont, dans un premier temps, au XIX ${ }^{\mathrm{e}}$ siècle, dispersés dans le Caucase ${ }^{4}$. Ainsi, le village de Sanda dans la région de Tsalka au sud de Tbilissi, en Géorgie non loin de la frontière arménienne, fut fondé entre 1835 et 1840 par des familles d'hellénophones originaires de la région montagneuse de Sanda (Trébizonde). Ils étaient allés s'installer dans cette partie de l'Anti-Caucase, non pas pour des raisons économiques, mais en vue de mieux conserver leur religion orthodoxe et leur langue. Ils obtinrent des autorités

4. Xanthopoulou-Kyriakou, 1994. 
russes des terres appartenant à l'État et s'établirent sur les ruines d'un ancien village géorgien. De 1843 à 1846, ils construisirent leur église dédiée au Saint-Esprit sur les ruines de l'ancienne église orthodoxe géorgienne, et, à partir de 1847, une école enseignant la langue et les lettres grecques commença à fonctionner dans l'église, sous la responsabilité du prêtre ${ }^{5}$.

Un peu plus tard après la guerre de Crimée, on a assisté à une nouvelle migration. En 1864, 96 familles grecques de l'arrière-pays montagneux de Trébizonde, la plupart originaires de Sanda, se sont installées provisoirement à Tsalka, puis à Tiflis, en attendant d'obtenir des terres achetées au prince Baratov dans la région de Tetri-Tskaro, voisine de Tsalka. Ils ont formé alors une communauté agraire de 5 villages : Ambeliani, Megali et Mikri Irangka, Ivanovka, Phtelen et Vizirovka. Le chef-lieu de ce petit territoire était Mikri Irangka ou Sekitli avec son église dédiée au prophète Élie (Profiti Ilias), dont la construction commencée en 1866 s'acheva en 1868. Les églises et les écoles qui leur étaient étroitement liées ont joué un rôle capital dans la préservation de l'identité de ces villages ${ }^{6}$. Plusieurs familles ont de nouveau migré après 1878 , comme d'autres groupes venant directement du Pont, vers la région de Kars qui était proche.

Le gouvernorat de Kars, annexé par la Russie de 1878 à $1920^{7}$, avait une population pluriethnique comprenant Turcs, Turkmènes, Kurdes, Karapaches et quelques Arméniens, Lezgines et Ossètes. Les Russes étaient peu nombreux, présents uniquement dans les villes. En 1918, les Grecs sujets de l'Empire russe y étaient au nombre de 58010 auxquels s'ajoutaient 8 à 10000 réfugiés Grecs pontiques venus du territoire ottoman, ce qui fait en tout 70000 Grecs, répartis dans 74 villages, sur une population totale d'environ 300000 habitants 8 .

5. Parallèlement, selon le même processus, des migrants provenant de la région d'Imera (non loin de Sanda) fondèrent dans cette même vallée de Tsalka un village du même nom, Imera, en 1835, construisant leur église dédiée à Saint Pierre et Saint Paul et le bâtiment d'une école attenant à l'église à partir de 1838-1840.

6. ANGELIDIS, 1999 et 2005.

7. Selon le traité de Berlin (1878), l'occupation russe du gouvernorat était octroyée pour 40 ans. Il n'était pas intégré aux structures territoriales de l'Empire, mais avait un statut d'administration militaire. Les infrastructures militaires et de transport furent construites par la Russie à cause de l'importance stratégique de la région pour la protection de la zone pétrolière de Bakou sur la Caspienne.

8. Ils étaient venus principalement du sud de la région pontique (Chaldia, Erzéroum, Kolonia, Sivas, Neokaisaria et Amasia) et de la vallée de Tsalka (actuellement en Géorgie) au cours de la période 1877-1884. Ils s'étaient d'abord installés dans des villages abandonnés par les Turcs, puis avaient formé des commissions chargées de trouver les 


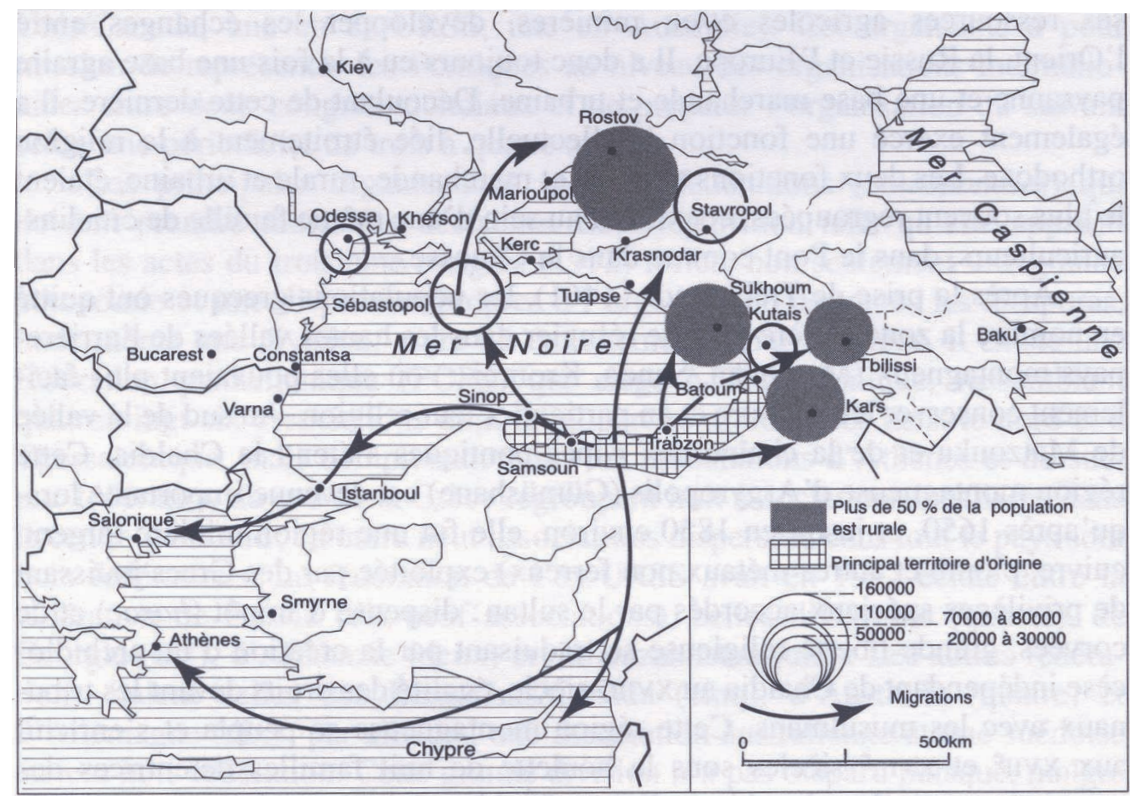

Source: Agtsidis V. (1991).

FIGURE 2. LES GRECS DE LA MER NOIRE DANS L'EMPIRE RUSSE (1914)

Bruneau, 1994, p. 205.

\section{Le ré-enracinement dans la « mère-patrie »}

Dans un second temps, les dispersions se sont faites en Grèce surtout à la suite de l'échange des populations (1923-1924). La spécificité du milieu géographique d'origine était tellement forte qu'elle a largement influencé les réfugiés dans le choix des sites de leur installation dans le Nord de la Grèce. Cependant l'échange forcé a entraîné la dispersion de ceux issus de telle ou telle petite région du Pont. «Quelques groupes d'échangés ont réussi à emporter les restes du saint tutélaire de leur bourgade, ou quelques vieux objets d'église dédiés à sa mémoire ${ }^{9} \gg$, ce qui leur permettra par la suite d'établir formellement un lien entre leur nouveau village reconstitué en Grèce et leur village d'origine. Les exemples de Sanda, Imera ou Kromni sont significatifs d'une telle situation.

sites les plus favorables pour l'installation et la construction de leurs nouveaux villages. Mavrogenis, 1963.

9. Société des Nations, 1926, p. 26. 
Beaucoup de Sandètes, par exemple, arrivèrent d'abord à Kalamaria, faubourg de Thessalonique, où ils sont restés en quarantaine, avant de pouvoir s'installer dans un village ou une ville de leur choix en Macédoine ou en Thrace. Soixante-dix familles originaires de Sanda décidèrent de construire leur propre village, Nea Sanda, à $30 \mathrm{~km}$ au Nord de Thessalonique. Ce bourg fit, dès cette époque, figure de métropole des Sandètes en Grèce, ses habitants étant particulièrement entreprenants ${ }^{10}$.

Cette dispersion aurait pu avoir pour conséquence la perte rapide, à la deuxième ou troisième génération, de ces identités territoriales ou leur absorption dans une identité plus large, celle de « réfugiés ». On constate en effet ce phénomène dans la vie associative de l'entre-deux-guerres, et même jusqu'aux années 1960. Mais, si on prend l'exemple de Kalamaria, quartier de Thessalonique marqué par l'installation massive de réfugiés parmi lesquels un grand nombre de Pontiques, on est frappé, dès les premières années (1923-1926), par la vigueur des associations ${ }^{11}$ pour qui la construction de l'école et de l'église était primordiale. Elles ont contribué à créer une identité commune à ces réfugiés encore en marge de la société grecque, et ont également à terme facilité leur intégration. La conservation des coutumes, de la langue, la transmission de l'identité liée au lieu d'origine n'était pas leur principal objectif, car celles-ci étaient alors vivantes au sein des familles. Ce besoin ne se fera sentir que bien plus tard dans les années 1970-1980 avec la deuxième et la troisième génération de descendants des réfugiés.

\section{Les Grecs pontiques en Australie}

Après la Seconde Guerre mondiale, l'occupation allemande et la guerre civile, les Grecs des zones rurales du Nord de la Grèce - dont des Pontiques - ont émigré pour des raisons économiques en Allemagne et dans quelques pays du Nouveau Monde (Canada, États-Unis, Australie). Une fois de plus, comme dans le Caucase, en Russie, en Macédoine ou en Thrace, ils ont dans leur majorité voulu rester fidèles à leurs racines historiques. On prendra l'exemple des Grecs pontiques d'Australie particulièrement représentatif de cette diaspora pontique du Nouveau Monde qui s'est formée après la Seconde Guerre mondiale.

10. L'exode rural vers Athènes et Thessalonique ou l'émigration en Allemagne ont, dans les années 1950-1960, vidé la plupart de ces villages de leur population. ChEImonidou, 1972 et Parcharidis, 1994.

11. Au cours des quinze premières années, dans ce quartier de 15000 habitants, ont été fondées 23 associations (IoANnidou, 1998, p. 144). Celles-ci, rassemblant les réfugiés sans distinction de lieu d'origine, avaient des objectifs sociaux, en vue de résoudre ou d'aider à résoudre des problèmes d'aménagement urbanistique et de soutenir les plus démunis. 
La migration qui a amené en Australie la plus grande partie des Pontiques australiens à partir de la Macédoine se situe entre 1952 et 1970. Ce furent environ 55000 migrants d'origine pontique qui s'installèrent en très large majorité dans les grandes villes, capitales des États de la Fédération australienne : 35000 à Melbourne, 8500 à Sidney, 6000 à Adélaïde, 5500 dans les autres capitales. Cet Hellénisme ne peut s'appréhender qu'à travers l'histoire des associations (somatia) et de leurs activités ${ }^{12}$. Le pôle principal est la seconde ville d'Australie, la plus industrielle, Melbourne, avec 35000 Pontiques sur 200000 Grecs. Quatre à cinq associations s'y sont créées à partir de la fin des années 1970 : Enosi Pontion Panagia Sumela, Pontiaki Estia, Enosi Pontion Panagia Sumela Whittlesea, Panpontiaki Kinotita Melvournis kai Victorias, Pontiaki Kinotita Melvournis kai Victorias, Syllogos Pontion Melvournis O Evxinos Pontos ${ }^{13}$.

Entre ces associations et à l'intérieur de certaines d'entre elles, des divergences voire des divisions n'ont cessé de s'exprimer alternant avec des périodes de concorde et même d'unité. Un très efficace comité de coordination entre les cinq associations de Melbourne pour l'organisation des cérémonies de commémoration du génocide (le 19 mai) a été fondé en 1998. Ces cérémonies ont eu un succès de plus en plus grand auprès de la communauté grecque et pontique, et ont obtenu une audience dans la société civile australienne en 2006. En 2007, les Arméniens et Assyro-Chaldéens se sont joints à cette commémoration qui est devenue commune aux Chrétiens d'Orient ${ }^{14}$. Les associations pontiques d'Australie ont également poursuivi des objectifs humanitaires dont le plus important, qui a concerné les Grecs réfugiés ou migrants, a eu une visée clairement nationale. Ce projet consistait à aider la population des Grecs de l'ex-URSS arrivés en Grèce à partir de 1989 à la suite de l'effondrement du régime soviétique ${ }^{15}$. C'est un

12. TAMIS, 1994, p. 107-198.

13. À partir du premier congrès mondial panpontique de 1985 , les diverses associations pontiques australiennes furent incitées à se regrouper en une Fédération (OPSA) qui fut créée en 1989. Malgré les divisions internes, cette fédération se réunit assez régulièrement et réussit à organiser une rencontre festive annuelle (Synapantema) des associations australiennes, en particulier de leurs troupes de danses, de leurs musiciens (lyrarides) et de leurs troupes théâtrales. Ces rencontres entre la totalité ou la majorité des associations pontiques australiennes sont très importantes pour l'établissement ou le renforcement des contacts entre jeunes.

14. TAMis, 1994, p. 131-148.

15. En 1990, un comité gréco-australien auquel participaient des représentants des trois associations de Melbourne a été constitué en vue d'aider les « rapatriés » (palinostountes) de l'ex-URSS à s'installer et à s'intégrer en Grèce. Ce comité a organisé diverses 
autre exemple de la façon dont fonctionnent les réseaux de solidarité dans l'espace transnational grec pontique entre l'Australie, la Grèce, la Russie et la Géorgie.

Une copie de l'icône de Panagia Sumela fut envoyée en Australie dans les environs de Melbourne en 1967. Depuis 1982, une cérémonie religieuse et une procession ont lieu chaque année le 15 août avec la participation de milliers de Pontiques australiens ${ }^{16}$. Un entrepreneur pontique originaire d'Armenochori (Florina), Onoufrios Gorozidis, qui émigra en Australie en 1954 et s'enrichit grâce à une chaîne de magasins de peinture, a acheté en 1996 une colline à $80 \mathrm{~km}$ au nord de Melbourne (comté de Yea) qu'il donna à la «Fondation pontique Panagia Sumela d'Australie et Nouvelle-Zélande » créée la même année ${ }^{17}$. Cette colline fut renommée Mont Mela ${ }^{18}$.

Les Grecs pontiques en Australie, mais aussi en Amérique du Nord ou en Allemagne, continuent de se référer à leur territoire et à leurs lieux d'origine à travers leurs associations et leurs églises. Les échanges culturels et religieux se font par le réseau national et transnational de ces institutions. Ils fonctionnent ainsi dans cet espace transnational reliant les communautés de la diaspora et celles de Grèce où ont parfois lieu des « retours » à partir du Caucase et de la Russie.

\section{Le « retour » des Grecs pontiques du Caucase en Grèce}

L'effondrement de l'URSS en 1990-1991 a entraîné l'arrivée en Grèce jusqu'en 2000 d'environ 200000 réfugiés-rapatriés (palinostountes) ${ }^{19}$. Depuis leurs villages du Caucase, les Pontiques ont souvent rejoint les villages d'installation en Macédoine des réfugiés de 1923 originaires des mêmes territoires dans le Pont : Sanda dans l'exemple étudié ici. Ainsi à Nea Sanda (Kilkís, Macédoine), ceux

manifestations culturelles en Australie s'adressant non seulement aux Pontiques, mais à l'ensemble des Grecs australiens de 1990 à 1992 (Kasapidis, 2007, p. 299-315).

16. Ce fut d'abord dans l'église Saint Nicholas de Yarraville puis dans la « Maison pontique » de Keilor. En 1978, la Fraternité pontique du Sud de l'Australie acheta une ferme à Birdwood (à $45 \mathrm{~km}$ d'Adélaïde) avec l'intention d'y construire un monastère dédié à la Panagia Sumela, mais ce projet resta sans suite et la ferme revendue 10 ans plus tard (TAMis, 1994, p. 192-197).

17. http://www.diaspora-net.org/psanz.html

18. La construction d'une colonie de vacances pour une centaine d'enfants et d'un sanctuaire ou monastère dédié à la Panagia Sumela est projetée sur ce terrain, qui est en cours de reboisement en oliviers et conifères. Un grand four pour barbecue a été aménagé, ce qui permet depuis 2003 un grand rassemblement annuel, festival de musique et danses pontiques et de nourriture grecque traditionnelle en novembre le jour de la Melbourne Cup.

19. VERGETI, 2003, p. 81. 


\section{CAHIERS BALKANIQUES}

venus de Géorgie (Tsalka et Tetri-Tskaro) ont construit à l'identique l'église du prophète Élie (Profiti Ilias) de Mikri Irangka (Sekitli). Le terrain a été offert par la commune, à proximité du bourg, près d'un bois de pins et sapins, au sommet d'une hauteur, avec une vue panoramique sur les collines rocheuses situées au nord de Nea Sanda. Les murs extérieurs de l'église sont revêtus de pierres de basalte vert foncé, provenant du village d'origine dans le Caucase comme l'icône du prophète Élie abritée dans le sanctuaire. Le toit et la croix perchée sur le clocher hexagonal sont en bronze ${ }^{20}$.

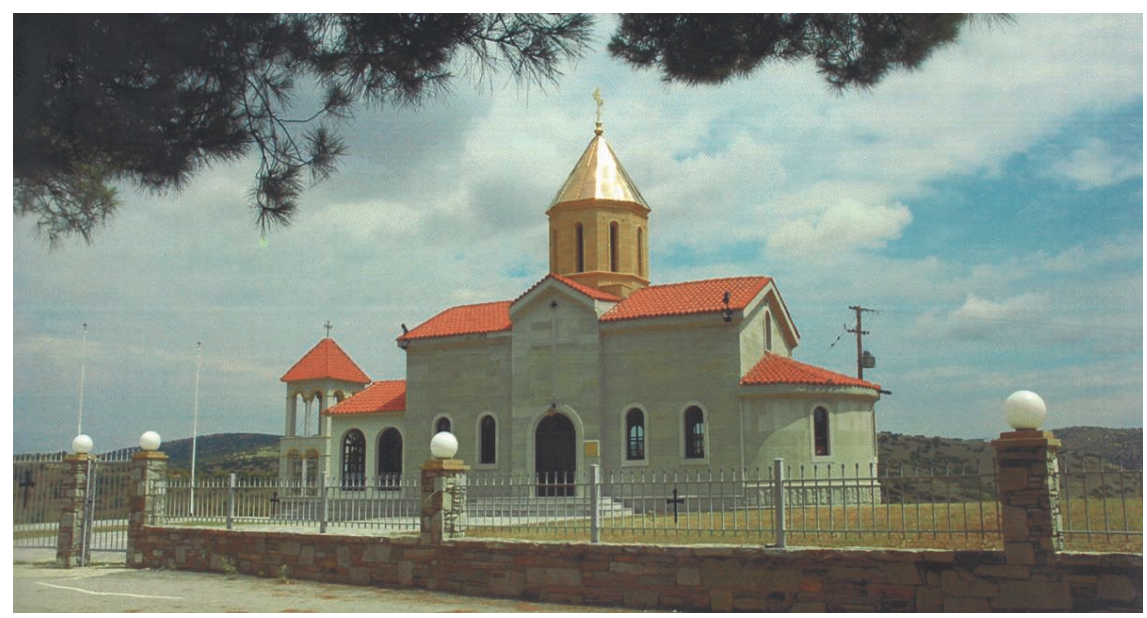

\section{FIGURE 3. ÉGLISE DE NEA SANDA}

Photo M. Bruneau

Ces migrations et ré-émigrations, ces déracinements et ré-enracinements successifs ont abouti à la création d'un espace-réseau diasporique, transnational,

20. Cette église construite en un an (1995) est l'œuvre d'un homme, Léonidas Papounidis, chirurgien dans l'ex-URSS devenu entrepreneur en Grèce, avec l'aide de quatre autres bienfaiteurs tous originaires de cette région de Tetri-Tskaro. C'est aussi l'œuvre d'une association créée à Thessalonique en 1995 «Enosi Pontion Pentakomi Sekitli», qui en 2005 regroupait 750 familles arrivées en Grèce pour la plupart entre 1991 et 1993 des cinq villages de Tetri-Tskaro. La fête du saint patron (panigiri) a lieu chaque année le 2 août, réunissant entre 2 et 3000 personnes venant de toute la Grèce, mais principalement de l'agglomération de Thessalonique. Cette communauté de réfugiés récents, dont une élite s'enrichit rapidement, a le projet de construire à côté de ce sanctuaire, le premier fondé en Grèce par des réfugiés récents de l'ex-URSS, un village reproduisant l'architecture de leurs villages caucasiens. 
à l'échelle mondiale, qui a une cohésion certaine. Celle-ci est à mettre en rapport avec une « iconographie », au sens gottmanien du terme ${ }^{21}$, qui facilite et même permet le maintien de liens au sein de cette diaspora.

\section{De l'icône de la Vierge de Sumela à l' « iconographie » grecque pontique}

L'icône de la Vierge de Sumela, provenant d'un monastère du Pont au sud de Trébizonde, par son histoire légendaire autant exceptionnelle que symbolique, est devenue le point focal de l'iconographie pontique et le symbole de leur unité. C'est autour d'elle que les Grecs pontiques en diaspora ont constitué leur « iconographie ». Héritiers directs des Byzantins, pour eux État, nation et orthodoxie sont étroitement liés. Panagia Sumela symbolise tout cela à la fois. Cette iconographie ne peut exister et s'exprimer pleinement qu'enracinée dans un haut lieu, le sanctuaire et le monastère de la Vierge de Sumela qui permet le grand rassemblement annuel qu'est le pèlerinage du 15 août. Sa fonction principale est d'assurer la reproduction de l'identité grecque pontique en exil et sa transmission d'une génération à l'autre. D'où la nécessité de le rendre possible partout où se trouve une partie importante de la diaspora, en Macédoine (Kastania), en Amérique (West Milford, New York), en Australie (Yea, Melbourne) ${ }^{22}$. L'icône de la Vierge de Sumela, installée dans son sanctuaire du Vermion dès 1952, a été le point de départ de la renaissance, ou même de la naissance de l'identité grecque pontique en diaspora. Le développement de la vie associative qu'on observe à partir des années 1970 a contribué à enrichir l'iconographie d'autres éléments, non religieux, soit culturels, soit politiques ou nationaux.

21. Le concept d'iconographie est emprunté à J. Gottmann (BRUNEAU, 2000): «L'iconographie est la somme des croyances, des symboles, des images, des idées, etc. dont une communauté a hérité et auxquels ses membres sont attachés. Ce lien entre les personnes et les "icônes" constitue le ciment du groupe et conduit à la définition d'un territoire dont les membres des autres communautés, attachés à d'autres iconographies, sont exclus » (GotTmann, 1955, II, p. 200). Cet « ensemble des symboles, abstraits et concrets, qui résument les croyances et les intérêts communs à une collectivité, constitue le ciment donnant sa cohésion et sa personnalité politique à cette collectivité ».

22. BRUNEAU, 2008. 
CAHIERS BALKANIQUES

286

La presse allophone dans les Balkans

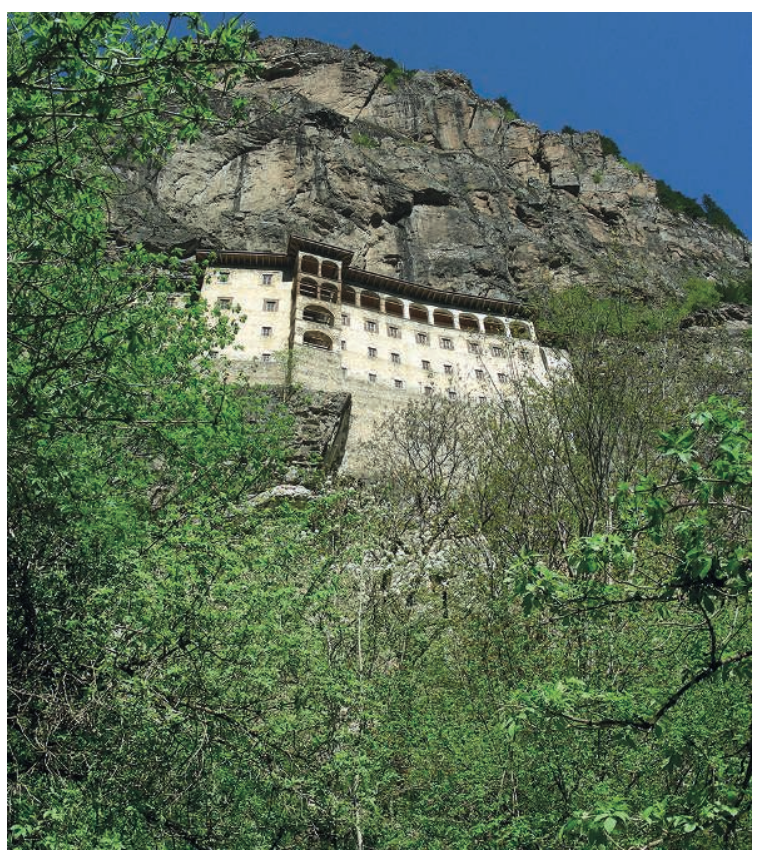

FIGURE 4. LE MONASTÈRE DE SUMELA

Wikimedia commons, photo Herbert Frank

Le personnage du résistant, armé jusqu'aux dents de poignards et de pistolets, dont les photos sont affichées et qui fait l'objet de représentations stylisées, est le motif iconographique le plus politique. Il s'élargit dans la référence au génocide (1919-1922) dont la reconnaissance internationale est revendiquée depuis le congrès panpontique de 1988. La journée du 19 mai 1919, date du débarquement de Kemal Atatürk à Samsun, a été choisie pour marquer le début du génocide. Elle a été, depuis 1994, officialisée par le Parlement d'Athènes pour des célébrations rappelant massacres, déportations, extermination de 350000 Grecs pontiques par les Turcs. Les photographies des massacres et déportations sont de plus en plus présentes dans les associations et même sur les murs des tavernes pontiques. Elles font maintenant partie de l'iconographie.

Celle-ci s'est également enrichie de deux autres éléments. L'aigle à une tête, emblème de l'Empire de Trébizonde et de la dynastie des Comnènes qui figurait sur les pièces de monnaie, est très largement placé en exergue dans la presse et les périodiques pontiques. Le slogan sur la Romania, extrait d'une chanson populaire, 
se rencontre par ailleurs en tête de bien des publications : «Même si la Romania est passée, elle refleurira et portera de nouveaux fruits ${ }^{23} \gg$.

Ainsi les Grecs pontiques descendants des Byzantins ont progressivement constitué une « iconographie » de peuple en diaspora. Cela est passé par la reconstitution de hauts lieux et de lieux de mémoire. Le processus ne s'est véritablement enclenché qu'à partir de la réinstallation de l'icône de la Vierge de Sumela en Macédoine. C'est à partir et autour de cet élément religieux très profond qu'une iconographie faite d'images et de symboles s'est diversifiée et enrichie. Le rassemblement du 15 août au sanctuaire de Panagia Sumela reste la manifestation annuelle la plus forte des Grecs pontiques dans le monde, manifestation identitaire et politique autant que religieuse.

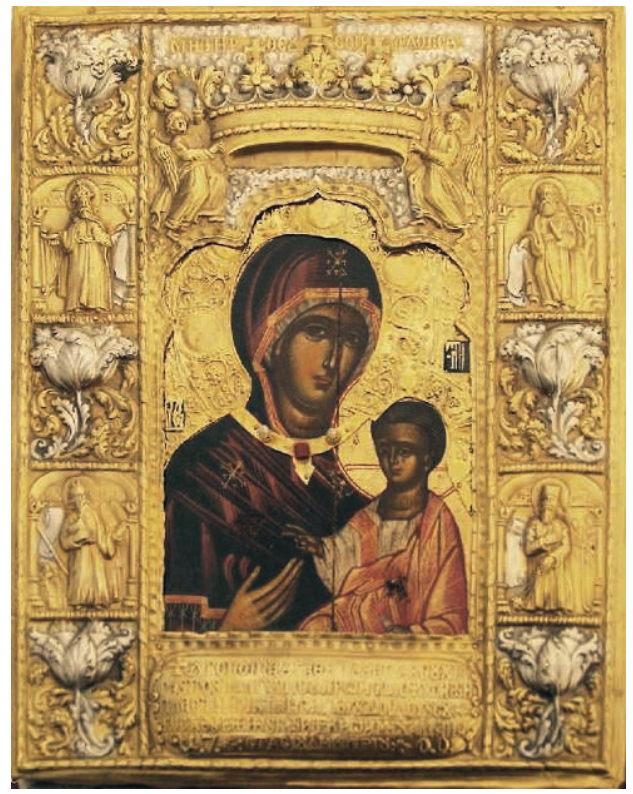

FIGURE 6. ICÔNE

\section{DE LA VIERGE DE SUMELA}

Il s'agit d'une copie à l'identique de l'icône originale. Celle-ci, déjà vieille de plus de 15 siècles, et gardée dans un bâtiment incendié, a été enterrée par les moines lors de leur départ en 1923 et l'est restée jusqu'en 1931 lorsqu'Atatürk les autorisa à venir la rechercher. Elle a beaucoup souffert et est très fragile.

23. Le terme de Romania qui désigne également l'Empire byzantin et celui de Trébizonde a été repris par les Grecs pontiques. Ils se le sont approprié (FANN, 1991). Des images ou photographies des principales églises de Trébizonde (Agios Eugenios, Agia Sophia) ou du lycée (le Frontistirio) dans lequel fut formée la plus grande partie de l'élite grecque pontique apparaissent souvent sur les murs des salles de réunion des associations ou dans les publications pontiques. 
CAHIERS BALKANIQUES

La presse allophone dans les Balkans

\section{Les congrès pontiques et le « génocide » revendiqué}

Les Grecs pontiques ont une tradition très ancienne d'organisation de congrès rassemblant la majorité des représentants de leur vie associative en Grèce, en Russie ou dans le monde (diaspora) ${ }^{24}$. Leurs premiers congrès internationaux furent organisés à la fin de la Première Guerre mondiale lorsque se posa le problème de la disparition de l'Empire ottoman et de la création d'une république indépendante du Pont ou d'un rattachement à la Grèce ${ }^{25}$.

Ces congrès eurent lieu surtout dans le Caucase ou au sud de la Russie où existaient de nombreuses communautés grecques pontiques (Tbilissi 1917, Taganrog 1917, Krasnodar 1917 et 1918, Bakou 1918, Batoum 1919). À Marseille (1918), Constantin Constantinidis réunit le premier congrès panpontique avec des délégués de plusieurs pays européens, d’Égypte, des États-Unis et de Grèce, mais sans représentants de Russie ou de Turquie qui en furent empêchés ; on y revendiqua pour la première fois l'indépendance d'une « république du Pont » avec l'envoi d'un télégramme à Trotski. Cependant le véritable premier grand congrès mondial panpontique n'est survenu qu'en 1985 à Thessalonique.

De 1985 à 2002, ont eu lieu ensuite, toujours à Thessalonique, cinq congrès mondiaux, auxquels ont participé des représentants de la très grande majorité des associations grecques pontiques de Grèce et du monde ainsi que des universitaires, des chercheurs et des journalistes. Les associations des pays de l'ex-URSS n'ont pu y participer qu'à partir du second (1988). Les trois premiers de ces congrès (1985, 1993, 1997) ont été inaugurés par le Premier ministre grec en personne (Papandreou puis Mitsotakis). À partir du second congrès, les associations se sont regroupées en fédérations dont le nombre et le périmètre a évolué en fonction des circonstances et des rivalités de personnalités. Ces congrès ont eu un double objectif scientifique (histoire, laographie, culture) et politique. On y discuta les modes d'organisation de l'Hellénisme pontique au niveau mondial et on y énonça les principales revendications adressées au gouvernement grec et à la communauté internationale.

24. Cette tradition a ses racines dans l'Antiquité, dans le Koinon ton Pontion qui à partir du $\mathrm{II}^{\mathrm{e}}$ siècle apr. J.-C. a rassemblé une dizaine de cités des côtes de la mer Noire du Pont et de Paphlagonie dans la cité de Amastris (Amastra dans la Turquie actuelle). Cette institution antique a été remise à l' honneur récemment par la fondation de Panagia Sumela qui à partir de 1964 a institué ce Koinon, comme une assemblée générale des Pontiques se réunissant tous les trois ans à l'occasion du pèlerinage annuel sur le site du nouveau monastère pour élire les membres du conseil d'administration de la fondation. Il traite essentiellement de questions religieuses, mais parfois aussi de sujets ayant trait aux intérêts nationaux des Pontiques et de l'Hellénisme.

25. KaÏsIDIS, 2007. 
D'un congrès à l'autre, on retrouve les mêmes thèmes qui n'ont pas toujours progressé26 ${ }^{26}$ Le premier et principal à partir de 1988 est le thème de la reconnaissance du génocide des Grecs pontiques (1916-1923) par l'État grec, l'État turc et les Nations unies. Les autres thèmes qui reviennent constamment sont : la présence de données sur l'histoire de l'hellénisme pontique dans les programmes et manuels des écoles primaires et secondaires en Grèce, l'organisation de séminaires et de chaires consacrés à l'Hellénisme d'Asie Mineure dans les universités de Grèce et d'ailleurs, l'aide aux réfugiés provenant des pays de l'ex-URSS en Grèce et aux communautés grecques présentes dans ces pays, la prise en compte des questions d'intérêt national pour les Grecs concernant Chypre ou la Macédoine ex-yougoslave, l'établissement de liens avec les hellénophones musulmans de Turquie, en particulier ceux de la région pontique (le droit à conserver leur langue, l'octroi de permis de séjour et de travail à ceux d'entre eux qui ont immigré en Grèce), la préservation et la reconnaissance des monuments grecs de l'Antiquité à l'époque contemporaine en Turquie.

Ces différentes manifestations, nombreuses et riches par-delà les divisions et conflits au sein des associations, ont beaucoup contribué à faire connaître tous ces thèmes au public grec d'origine pontique ou non. Elles ont presque toutes donné lieu à publication. Elles sont un des principaux facteurs de la vigueur de l'affirmation identitaire des Pontiques en Grèce et dans le monde.

\section{Les représentations cartographiques du territoire du Pont revendiqué, leur place dans l'iconographie pontique}

Les associations de Grecs pontiques en Grèce et dans le monde ont toutes fait une place importante dans leur iconographie, qui sert de base à la transmission de l'identité, aux représentations cartographiques du territoire du Pont, composante de l'actuelle Turquie. À l'origine de ces cartes se trouve la revendication de la création «d'une République indépendante comprenant une partie de l'ancien royaume de Trébizonde des Comnènes, depuis la Caucasie jusqu'à l'ouest de Sinope, avec son hinterland » telle que définie par Socrate CEconomos et Constantin Constantinides dans le Mémoire présenté à la Conférence de la Paix par les Délégués du Pont-Euxin en février 1919. Dès novembre 1917 avait circulé sous la forme d'une carte postale une « carte de la République du Pont » au $1: 350000^{\mathrm{e}}$, distribuée au premier congrès mondial des Pontiques qui s'était tenu à Marseille du 22 janvier au 4 février 1918 à l'initiative et sous la présidence de C. G. Constantinidis. Au bas de la carte figurait le slogan « Citoyens du Pont-Euxin, levez-vous ! Rappelez aux nations libérales vos droits suprêmes

26. KAÏSIDIS, 2007. 
à la Vie et à l'Indépendance ». Cette carte du territoire revendiqué pour une république du Pont entre 1918 et 1920 a été reprise et diffusée par un grand nombre d'associations, en commençant par le Comité des Études pontiques (Epitropi Pontiakon Meleton). Elle est devenue emblématique, c'est une icône essentielle de la diaspora des Grecs pontiques en Grèce et dans le monde entier.

\section{La lyre, la musique et les danses pontiques, marqueurs identitaires}

La lyre (lyra en grec, kemençe en turc) et la musique sont devenues à travers la vie associative un marqueur essentiel de l'identité pontique en Grèce et dans le monde. La musique accompagne les chansons populaires (dimotika tragoudia) ou épiques (akritika) ${ }^{27}$ et très souvent les danses, le joueur de lyre (lyraris) se tenant debout au milieu du cercle des danseurs ${ }^{28}$. Elle est l'instrument principal, mais elle peut être accompagnée d'un gros tambour (davul) et d'un hautbois (zurna) ou d'une clarinette (klarino) pour les danses traditionnelles ${ }^{29}$.

Les danses ne sont pas toujours accompagnées de chants. Elles sont vivantes, très rythmées et se distinguent par leur style plus que par leurs pas ${ }^{30}$. La danse n'est pas seulement un moyen d'expression, mais une façon de vivre. Elle permet spontanément au peuple pontique de vivre collectivement ses moments de joie comme de malheur. Création populaire renouvelée tout au long de son histoire, elle est le plus puissant trait culturel de ce peuple ${ }^{31}$. Après l'échange des populations (1923), elle est devenue un moyen essentiel d'assurer la survie, la conservation et la transmission de l'identité pontique. Les danses collectives sont cultivées et enseignées par la plupart des associations comme un marqueur identitaire très fort et assez facile à transmettre aux jeunes. Des concours de danse et de musique ont lieu chaque année à plusieurs reprises, au cours de rassemblements spécifiques en Grèce et à l'extérieur, en particulier en Allemagne ou à l'occasion du pèlerinage à Panagia Sumela à Kastania (Macédoine) ; ils permettent de réunir des foules de tous âges et de les faire communier dans la célébration de leur identité pontique.

27. TAMIS, 1982.

28. Chairopoulou, 1994.

29. Le joueur de lyre, qui très fréquemment est aussi chanteur, joue un rôle essentiel dans l'animation d'une fête que ce soit un mariage ou toute autre occasion festive. Il accompagne également le mort jusqu'à sa dernière demeure au cimetière.

30. ZOURNATZIDIS, 2008.

31. Athanasiadis, 1986. 


\section{Le dialecte pontique et le théâtre, objets identitaires}

La spécificité de la langue est avec celle du territoire un élément fondamental pour définir le peuple grec pontique. Comme d'autres dialectes grecs, le « pontique » découvert à la fin du $\mathrm{XIX}^{\mathrm{e}}$ siècle est resté jusqu'aux années 1930 une langue de tradition orale. Il a vécu en contact de longue durée avec les autres langues du milieu pluriethnique auquel ont appartenu les Pontiques : arménien, kurde, langues kartvèles (laze, géorgien), turc. Issu comme tous les autres dialectes de la koinè hellénistique, il a comme le cappadocien évolué indépendamment de ceux-ci, car il en a été séparé géographiquement depuis le $\mathrm{XI}^{\mathrm{e}}$ siècle par les invasions seldjoukides ${ }^{32}$. La ponticophonie est encore présente à l'époque contemporaine dans des groupes de religion grecque orthodoxe, protestante (depuis le $\mathrm{XIX}^{\mathrm{e}}$ siècle), ou musulmane (régions de Tonya et de Of-Sürmene). En diaspora, le dialecte pontique s'est conservé comme langue parlée grâce aux habitats ruraux ou semi-ruraux de Thrace et de Macédoine et aux îlots ponticophones de Russie et de Géorgie.

En Grèce, après l'échange de 1923, les Pontiques ont considéré dans un premier temps que leur langue était promise à une disparition inéluctable. Malgré tout, le dialecte pontique résiste à sa disparition prévue, bien qu'il ne soit pas enseigné dans les collèges ou les lycées, mais seulement dans certaines associations. L'arrivée des réfugiés pontiques de l'ex-URSS depuis les années 1990 et l'immigration de travailleurs ponticophones musulmans de Turquie en Grèce et dans la diaspora lui ont redonné vie comme langue de communication ${ }^{33}$.

C'est dans les représentations théâtrales données régulièrement en Grèce par des troupes d'acteurs amateurs que le dialecte pontique continue à faire communier dans leur culture un large public de descendants des réfugiés, même ceux qui n'ont qu'une connaissance passive de ce dialecte. Il fait revivre la société des bords de la mer Noire de la fin du XIX ${ }^{\mathrm{e}}$ et du début du $\mathrm{XX}^{\mathrm{e}}$ siècle en costumes de l'époque. Il s'agit d'un véritable rituel, les représentations se donnant le plus souvent lors de fêtes religieuses ${ }^{34}$. Le théâtre, en Australie comme en Grèce, est l'une des meilleures façons de transmettre la langue aux jeunes générations ${ }^{35}$. Comme les

\section{Drettas, 1998.}

33. Des poèmes, des contes, des proverbes sont régulièrement publiés dans des revues telles que Pontiaki Estia ou Archeion Pontou.

34. FANN, 1991.

35. Ce théâtre qui s'est développé en Grèce surtout après 1949 se réfère aussi à la vie des réfugiés déracinés (xeniteas), en situation liminale ou marginale dans la société grecque. Créé et joué pour un public pontique, il transmet une culture traditionnelle tournée vers le passé, vers la patrie perdue reconstituée le temps de la représentation. Il est fondé sur le 
autres éléments de l'iconographie, il sert à établir et définir des frontières autour de la « ponticité » afin de perpétuer l'identité ethnique des Pontiques au sein de la société grecque qui exerce sur eux une très forte pression assimilatrice ${ }^{36}$.

Ayant perdu leur territoire d'origine, leur « patrie », les Grecs pontiques en ont fait une construction symbolique, idéelle, basée sur la distinction des nôtres (t'emeter) à l'intérieur de la communauté et des autres, les étrangers (xen) à l'extérieur. Le théâtre est le lieu par excellence où, l'instant d'une représentation, la « patrie » se reconstitue par une communion entre la scène et la salle ${ }^{37}$.

\section{Les monuments porteurs de l'iconographie pontique et d'un récit de l'bistoire-mémoire}

Depuis la reconnaissance officielle en 1994 par le Parlement grec du génocide des Grecs pontiques et de la journée du 19 mai pour sa commémoration, ceux-ci ont construit dans les espaces publics de chaque ville, et même souvent dans les villages habités par des descendants des réfugiés, des monuments dédiés à l'Hellénisme pontique et/ou au « génocide ». On trouve, de façon récurrente, dans tous ces monuments commémoratifs des éléments de l'iconographie pontique, en dehors même du Héros ou Résistant pontique (Antartis), omniprésent. On a le plus souvent une combinaison de plusieurs éléments de cette iconographie ${ }^{38}$.

Ces monuments sont dus avant tout à l'action de leurs associations et des collectivités territoriales, mais aussi à l'appui décisif sur le plan financier de l'État grec (d'avant la crise) qui a reconnu, soixante-dix ans après le déracinement, le droit au souvenir et le devoir de mémoire. Ils sont, par leur présence et par les cérémonies commémoratives qu'ils permettent une ou deux fois par an, la manifestation visible aux yeux de la population de ce droit au souvenir et aussi à la différence. Ces monuments ont donc une double fonction de transmission de la mémoire et de l'identité des Grecs réfugiés au sein de la « mère-patrie » grecque.

mythe de la mort et de la résurrection du Pont et de sa société, le distique de la Romania extrait d'une chanson populaire et mis en exergue par de nombreuses associations et media pontiques.

\section{Fann Bouteneff, 2002.}

37. Le théâtre utilise le dialecte pontique, met en scène la culture traditionnelle sous toutes ses formes (musique, danses, costumes, chansons populaires) évoque l'histoire, l'exil (xeniteia). La maison familiale, le village d'origine, et reconstitué après l'exil en Grèce, les associations sont le contenant de cette culture identitaire qu'il faut à tout prix préserver. Ils permettent de dresser les frontières de la « ponticité » en Grèce et dans le monde tandis que la grécité du Pont, le territoire d'origine, est perdue, en grande partie effacée à la suite de l'Échange des populations (1923).

38. Bruneau, Papoulidis, 2004. 
Des éléments iconographiques pontiques précédemment décrits sont donnés en plus ou moins grand nombre dans ces monuments sans qu'ils soient obligatoirement mis en ordre chronologique ou même logique. Cependant les bas-reliefs se présentent comme un récit de l'histoire des Grecs pontiques, à partir de l'expédition mythique des Argonautes à la recherche de la Toison d'Or. Le seul monument qui comprend la totalité de ce récit, ou plutôt de ce méta-récit avec tous les symboles iconographiques sur une vaste fresque sculptée en bas-relief est celui de Kastoria. C'est aussi le plus politique, puisqu'il comporte également un buste de Kapetanidis, journaliste nationaliste, au-dessus d'une carte du « territoire du Pont revendiqué (1918-1922) ». Ce monument récent (2001) représente un cas extrême dans le corpus existant ${ }^{39}$.

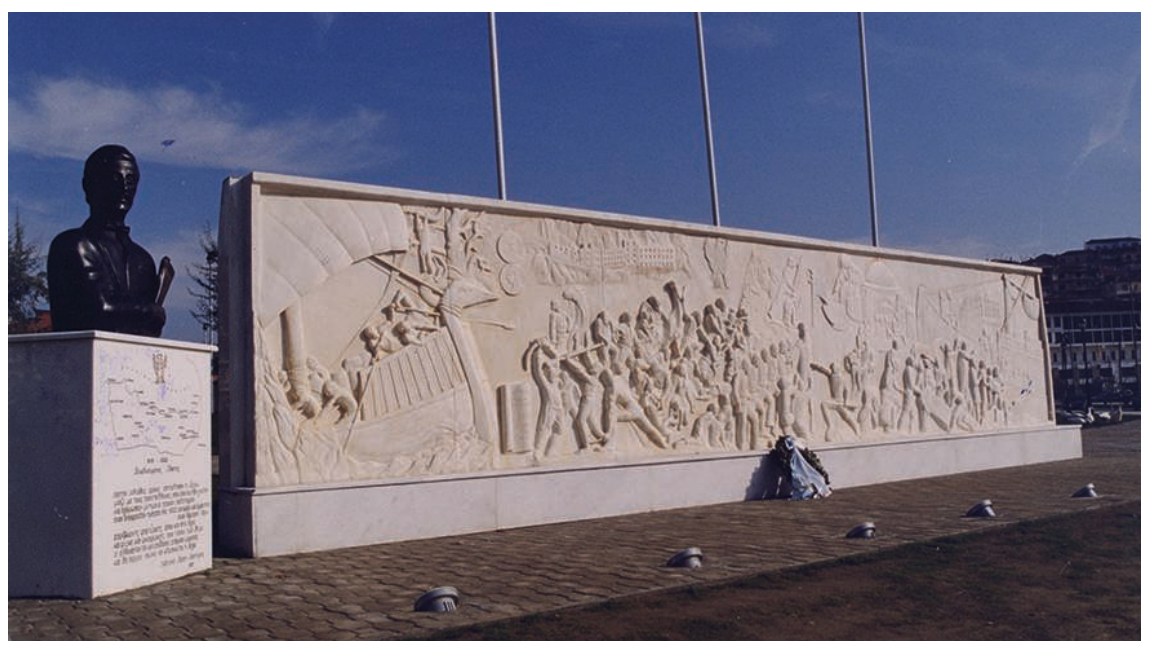

FIGURE 5. LE MONUMENT DE KASTORIA

Photo M. Bruneau

Les monuments commémoratifs sont, à côté des périodiques associatifs, de la littérature historique et des récits de témoins, l'un des porteurs essentiels de ce

39. Le monument aussi récent de Agia Varvara (2002), banlieue d'Athènes, représente une façon beaucoup plus distanciée, et non nationaliste, de traiter la mémoire pontique, à partir uniquement de symboles surtout religieux (croix byzantine, porte de Panagia Sumela, candélabre) et culturels (lyre), le fusil et le sabre étant les seuls signes de la résistance et du conflit armé. La porte est ouverte sur l'avenir, sans allusion à un « retour » éventuel. Deux autres monuments (Florina, Aspropyrgo) présentent même la lutte armée des résistants pontiques comme un fait du passé désormais révolu, le thème central étant l'avenir. 
que P. Ricœur (1985) appelle « l'identité narrative », liée à une mise en récit de la communauté. Les Pontiques ont en effet le corpus iconographique et identitaire le plus riche qui se différencie nettement de celui des autres réfugiés. Il se nourrit de leur mémoire et l'alimente en même temps ; il permet la construction d'un métarécit. Bien qu'ils ne soient pas statistiquement les plus nombreux, ils dominent la scène mémorielle des réfugiés d'Asie Mineure et de Thrace orientale.

Le droit à la mémoire (Dikaioma stin Mnimi) s'affirme par les musées, les expositions et les voyages-pèlerinages dans les patries « inoubliables ». Les Pontiques ont construit leur propre centre : le « Conservatoire des objets et souvenirs de l'Hellénisme du Pont » (Stegi Keimilion tou Ellinismou tou Pontou), inauguré en 1988 dans un quartier de Nea Smyrni, rue des Martyrs inconnus. Leur association Epitropi Pontiakon Meleton, fondée dès 1927, avait pour objectif : « le recueil, l'étude et la publication de matériaux sur la langue, les coutumes (laographie) et l'histoire du Pont ». Dans ce but, l'association a créé une revue d'un niveau scientifique international, Archeion Pontou, qui n'a pas cessé de paraître de 1928 à nos jours (48 tomes publiés et 18 livres séparés). Le bâtiment de trois étages abrite non seulement une bibliothèque, des salles de réunion et de séminaires, mais aussi depuis 1999 un « Musée de l'Hellénisme Pontique », un musée ethnographique, retraçant la vie et montrant les principaux produits de l'art et de l'artisanat de cette société qui a cessé d'exister dans son territoire d'origine en $1923^{40}$.

Les Pontiques en la personne de Konstantínos Fotiadis, connu comme l'historien du génocide, ont organisé une exposition itinérante « Le Pont : Droit à la Mémoire » de documents, de livres, d'images et de photographies sur l'Hellénisme du Pont et le génocide des Grecs pontiques ${ }^{41}$. Inaugurée en mars 2005 à Thessalonique, elle a été ensuite présentée à Véroia, Dráma, Kavala, Volos, Ptolemaïda, Acharnon, dans la Technopole du Dème d'Athènes et dans la station de métro de la place Syntagma (2016). Elle a également circulé dans plusieurs villes de Russie et d'Ukraine. En 2010, elle a aussi été organisée dans des villes d'Australie. Deux publications destinées à un grand public en présentent les différents thèmes et documents, abondamment illustrées en couleurs ${ }^{42}$. En mai 2018, une exposition plus importante accompagnée de nombreuses

40. Epitropi Pontiakon Meleton, 1999.

41. Fotiadis, 2005.

42. Fotiadis, 2005, 2010. 
conférences et concerts ou spectacles a été présentée au centre de Thessalonique en mettant l'accent là aussi sur le droit et le devoir de mémoire ${ }^{43}$.

À partir des années 1980, les réfugiés d'Asie Mineure, en particulier Pontiques, et leurs descendants, ont effectué des voyages-pèlerinages en car dans leurs territoires d'origine (Turquie) à partir de Thessalonique ou d'Athènes ${ }^{44}$. Ils cherchent à retrouver la trace de leur maison ou de celle de leurs ancêtres, à connaître de visu le village ou la petite ville de leurs origines. Ils effectuent également une visite-pèlerinage aux monastères, en particulier à celui de Panagia Sumela. Ils prennent conscience de l'évolution de ce territoire et de sa population à très forte identité musulmane et turque, même hellénophone. La culture de la vie quotidienne a de nombreux points communs avec celle dont ils ont hérité (cuisine en particulier), de même la musique et les danses. Ils constatent que la plupart de leurs anciennes églises sont devenues des mosquées, plus rarement des musées et que leurs anciennes écoles d'architecture néoclassique sont toujours des écoles, turques évidemment ${ }^{45}$.

\section{Conclusion : des frontières à la diaspora}

Avant de devenir une diaspora mondiale, les Grecs pontiques ont été un peuple des frontières vivant aux marges et sur les marches de grands empires multiethniques (l'Empire romain, byzantin, les Empires ottoman et russe) puis de l'État-nation grec au contact des Balkans. Peuple des frontières dans le double sens du terme (limites entre États et fronts pionniers), ils ont construit par étapes un espace transnational réticulaire, une diaspora reliant diverses communautés de réfugiés et de migrants. Leur iconographie très riche leur permet de construire un territoire imaginaire se référant à leur territoire d'origine grâce à l'aménagement de lieux de mémoire (églises, monastères, monuments), qui sont autant de points d'appui

43. Les associations pontiques du monde entier ont également leurs sites internet qui vulgarisent en textes et en images les thèmes majeurs deleur culture et deleurs revendications concernant la reconnaissance du génocide. Quelques-unes ont également un périodique historico-culturel et informatif sur leurs activités et manifestations. Le mensuel Evxinos Pontos (219 numéros publiés à Thessalonique, avril 2018) de Ioannis Petalidis rend régulièrement compte de la vie associative et culturelle des Pontiques dans le monde, et publie des articles de fond sur leur histoire et leur culture. Fotiadis, 2018, p. 12-14.

44. Drettas, 2004.

45. Ils prennent beaucoup de photos, des films au caméscope, seul moyen pour eux de se réapproprier symboliquement ces paysages urbains ou naturels perdus. Les églises mosquées, musées, ou celles plus souvent en ruines, sont avec les écoles les principaux points de repère concrétisant le lien avec un passé irrévocablement révolu. 
pour reproduire et conserver au sein de leur territoire d'accueil leur identité distincte, mais étroitement liée à l'Hellénisme et à l'État-nation grec. Leur situation « acritique », de gardiens aux extrémités de territoires impériaux ou nationaux, n’a pas cessé de se reproduire, spontanément ou sous la contrainte, tout au long de leur histoire : frontière orientale byzantine, Caucase russe, Asie centrale, frontière nord de la Grèce. Le milieu montagneux de leurs origines les a très tôt amenés à la mobilité, aux migrations à la recherche de revenus complémentaires. Cette circulation migratoire entre Pont, Caucase, Russie, côtes de la mer Noire, les a prédisposés à la constitution d'une diaspora transnationale. Leur attachement à leur territoire d'origine, et même à leur terroir, passe par la religion chrétienne orthodoxe héritée de leur passé byzantin (églises, monastères, icônes) qui les différenciait de la majorité musulmane ottomane. Cette confrontation dans la longue durée avec une autre religion dominante, l'islam, et avec une autre langue elle aussi dominante, le turc, qu'ils ont parfois adoptée, a sans doute renforcé la vigueur de leur affirmation identitaire. Ces lieux et territoires de la mémoire, matériels ou immatériels, sont un substitut du territoire et des lieux d'origine perdus mais proclamés « inoubliables ». Ils sont les marqueurs d'une identité toujours vivante chez ceux qui n'ont pas connu leur patrie d'origine. Ils font de l'Hellénisme pontique une partie de l'Hellénisme qui a tenu à maintenir sa spécificité.

\section{Bibliographie}

\section{Monographies}

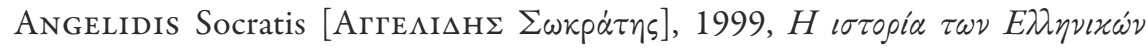

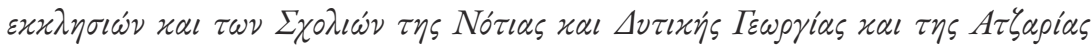
[l'histoire des églises grecques et des écoles du Sud et de l'Ouest de la Géorgie et de l'Adjarie] $\sum \omega \mu \alpha \tau \varepsilon i o$ П $\alpha \nu \alpha \gamma i \dot{\alpha}$ $\Sigma o v \mu \varepsilon \lambda \dot{\alpha}$ [Association Panagia Sumela], $\Theta \varepsilon \sigma \sigma \alpha \lambda$ ovikn [Thessalonique], $347 \mathrm{p}$.

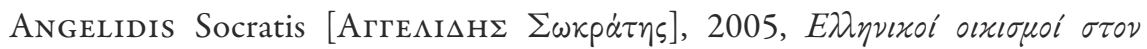

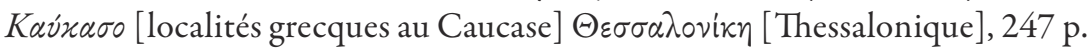

Bruneau Michel, Papoulidis Kyriakos, 2004, La mémoire de l'Hellénisme réfugié : les monuments commémoratifs en Grèce (1936-2004), Kyriakidis, Thessalonique, édition bilingue grec-français, $132 \mathrm{p}$. 
BRUneAU Michel (dir.), 1998, Les Grecs pontiques : Diaspora, identité, territoires, CNRS-Éditions, Paris, 250 p.

Bruneau Michel,2015,Del'Asie Mineureà la Turquie:Minorités, homogénéisation ethno-nationale, diasporas, CNRS-Éditions, Paris, 412 p.

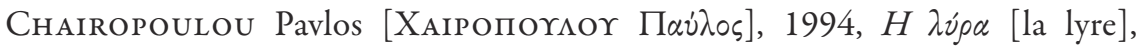

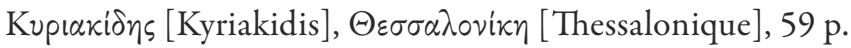

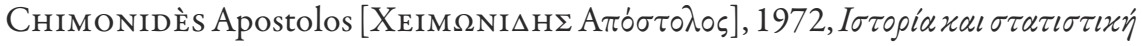

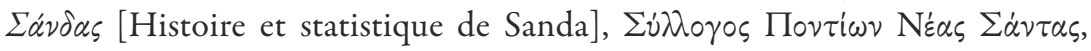
[Association des Pontiques de Nea Sanda, Kilkís], Kı $\lambda$ kis [Kilkis], 191 p.

Constantinides C. G., OEconomos S., 1919, Le Pont-Euxin devant le Congrès de la Paix, Mémoire présenté à la Conférence de la Paix par les Délégués du PontEuxin, Paris, 7 p.

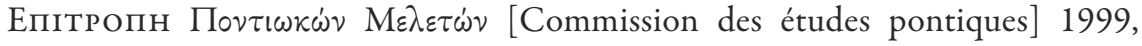

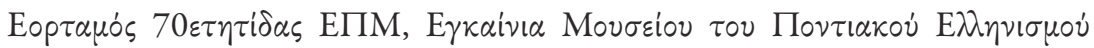
[Célébration du $70^{\mathrm{e}}$ anniversaire EPM, inauguration du musée de l'hellénisme

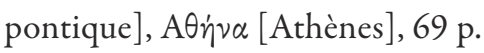

Fann Bouteneff Patricia, 2002, Exiles on Stage: the Modern Pontic Theater in

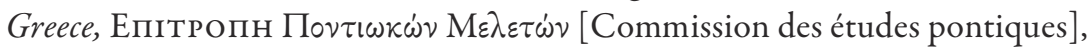
A $\theta \dot{\eta} v \alpha$ [Athènes], $243 \mathrm{p}$.

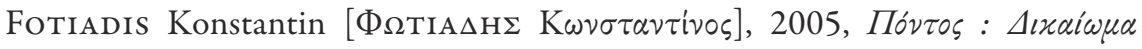

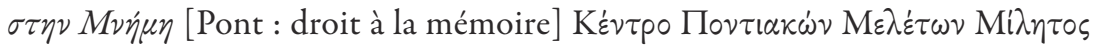

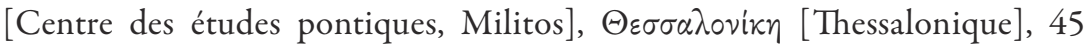
thèmes-panneaux.

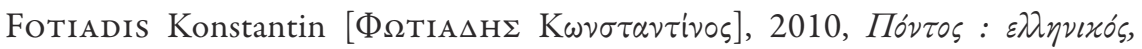

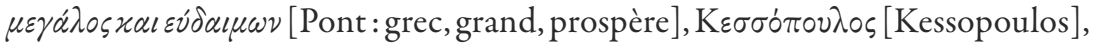
$\Theta \varepsilon \sigma \sigma \alpha \lambda \circ \nu i k \eta$ [Thessalonique], $221 \mathrm{p}$.

Gottmann Jean, 1955, La Politique des États et leur géographie, Armand Colin, Paris, 228 p. 


\section{CAHIERS BALKANIQUES}

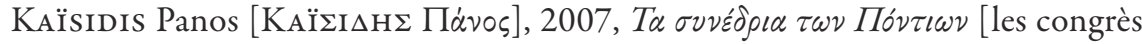

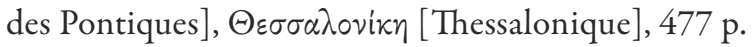

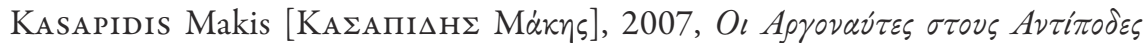

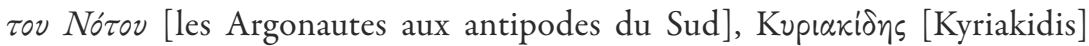
$\Theta \varepsilon \sigma \sigma \alpha \lambda$ ovikn [Thessalonique], $444 \mathrm{p}$.

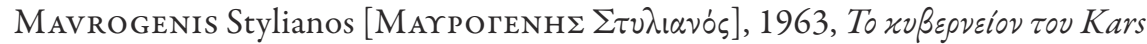

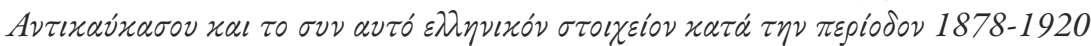
[le gouvernorat de Kars de l'Anti-Caucase et l'élément grec pendant la

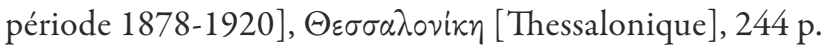

Meeker Mickael, 2002, A Nation of Empire: The ottoman legacy of turkish modernity, University of California Press, Berkeley, 420 p.

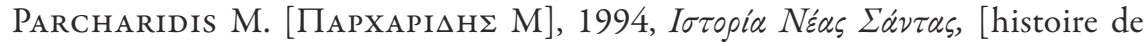
Nea Sanda], Kilkís [Kilkis], non publié.

Tamis Anastasios Myrodis, 1994, The immigration and settlement of Makedonian Greeks in Australia, La Trobe University Press, Melbourne, 387 p.

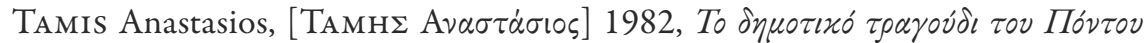

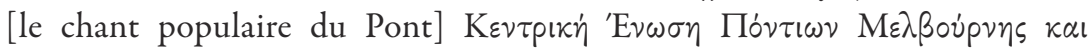

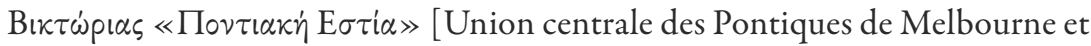
de Victoria, « Foyer pontique »], 48 p.

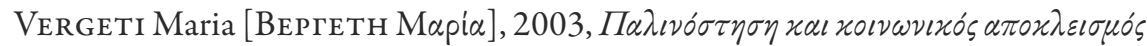

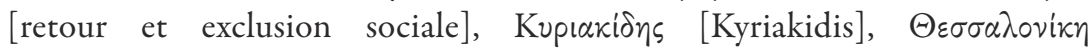
[Thessalonique], $94 \mathrm{p}$.

\section{Articles et contributions à des ouvrages}

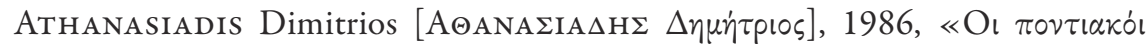

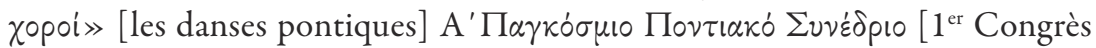

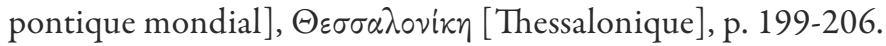

Bruneau Michel, 1994, « Territoires de la diaspora pontique », Espace géographique, DOI : 10.3406/spgeo.1994.3300. 
Bruneau Michel, 1995, «Lieux de mémoire, hauts lieux et diaspora : Sanda et Sumela dans la diaspora grecque pontique », l'Espace Géographique, 2, p. 124-134.

Bruneau Michel, 2000, « De l'icône à l'iconographie, du religieux au politique, réflexions sur l'origine byzantine d'un concept gottmanien », Annales de Géographie, 616, p. 563-579.

Bruneau Michel, 2002, « Hellénisme, Hellinismos : nation sans territoire ou idéologie ? », Géocarrefour: Revue de géographie de Lyon, 77 (4), p. 319-328.

BRUneau Michel, 2008, «Des icônes aux églises et aux monastères reconstruits par les réfugiés grecs d'Asie Mineure sur les lieux de leur exil », Diasporas Histoire et Sociétés, 12, p. 24-44.

Bryer Anthony, 1976, "The Pontic revival and the new Greece", in BRYER Anthony, Hellenism and the first geek war of liberation (1821-1830), Institute for Balkan Studies, Thessalonique, p. 171-190.

DretTas Georges, 1998, « La langue pontique comme objet identitaire : questions de représentations », in BRUNEAU Michel (dir.), Les Grecspontiques: diaspora, identité, territoires, CNRS-Éditions, Paris, p. 71-88.

Drettas Georges, 2004, « Modes et fonctions du voyage de pèlerinage des Gréco-pontiques en Turquie orientale », Cahiers balkaniques, 33, Inalco, Paris, p. 77-96.

FAnN Patricia, 1991, "The Pontic myth of homeland: cultural expressions of nationalism and ethnicism in Pontos and Greece, 1870-1990", Journal of Refugee Studies, 4 (4), p. 340-356.

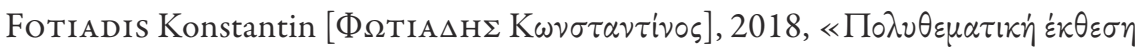

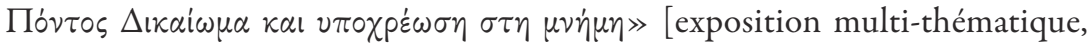

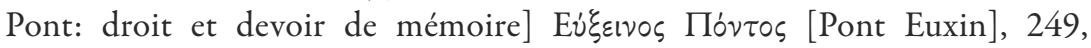
avril 2018, p. 12-14.

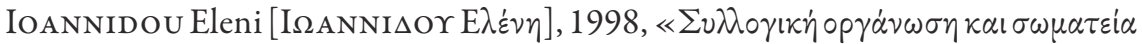

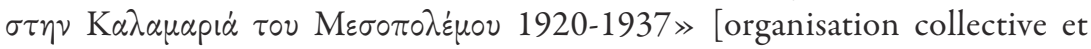
associations à Kalamaria dans l'entre-deux-guerres 1920-1937] I $\sigma \tau o p$ 


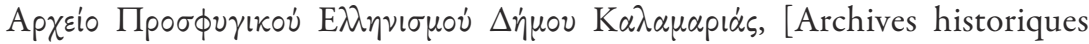

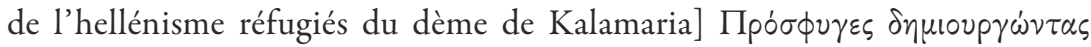

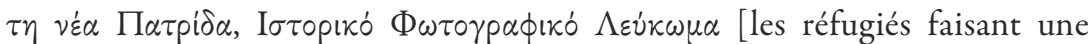
nouvelle patrie, album historico-photographique] University Studio Press, $\Theta \varepsilon \sigma \sigma \alpha \lambda \circ v i k \eta$ [Thessalonique], p. 142-160.

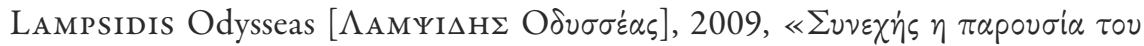

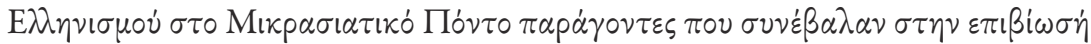
$\tau \circ v \gg$ [Présence continue de l'hellénisme dans le Pont micrasiate : les facteurs

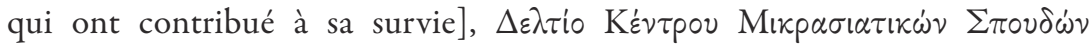
[Bulletin du centre d'études micrasiates], XvI, p. 25-53.

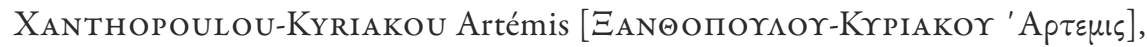

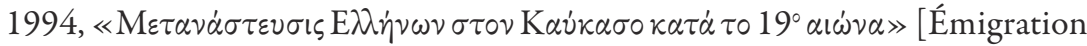

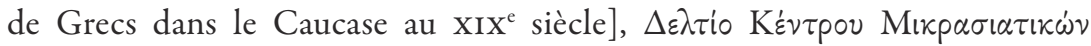
$\sum \pi \circ \nu \delta \omega^{2} \nu$ [Bulletin du centre d'études micrasiates], p. 91-172.

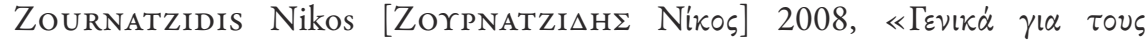

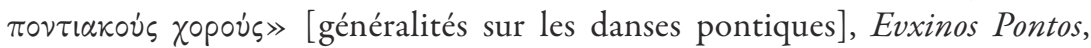
140, p. 14-16.

Résumé : les Grecs pontiques ont au sein de l'Hellénisme une identité très forte liée à la singularité de leur territoire d'origine qu'ils ont dû quitter lors de l'échange des populations édicté par le traité de Lausanne (1923). Réfugiés, vivant depuis lors en diaspora en Grèce et dans le monde (Russie, Amérique, Allemagne, Australie), ils ont constitué un espace-réseau transnational dans lequel ils ont aménagé des lieux de mémoire (églises, monastères, monuments commémoratifs) porteurs d'une iconographie très riche. Leurs réseaux associatifs actifs (fédérations, congrès) assurent la transmission de leur identité fondée sur une continuité de leur histoire depuis l'Empire byzantin de Trébizonde et, depuis 1988, sur la revendication de la reconnaissance du « génocide » (1919-1923) dont ils ont été victimes. Le héros pontique, l'icône de Panagia Sumela, la lyre, les danses et leur langue (le dialecte pontique) qui s'exprime à travers les chansons et le théâtre, sont les points forts de cette iconographie support de leur revendication d'un « droit et d'un devoir de mémoire ».

Mots-clefs : diaspora, hellénisme, iconographie, mémoire, Pont-Euxin, réfugié, réseau transnational 
Abstract: The Pontic Greeks have within the Hellenism a very strong identity related to the singularity of their territory of origin which they had to leave during the exchange of the populations enacted by the treaty of Lausanne (1923). Refugees, alive since then as a diaspora in Greece and in the world (Russia, America, Germany, Australia), they constituted a transnational space-network in which they arranged places of memory (churches, monasteries, memorials) carrying a very rich iconography. Their active associative networks (federations, congresses) ensure the transmission of their identity based on a continuity of their history since the Byzantine empire of Trebizond and, since 1888, on the claim of the recognition of the "genocide" (1919-1923) of which they were victims. The pontic hero, the icon of Panagia Sumela, the lyre, the dances and their language (the pontic dialect) which is expressed through the songs and the theatre, are the strong points of this iconography support of their claim of "a right and a duty to remember".

Keywords: diaspora, hellenism, iconography, memory, Pont-Euxin, refugee, transnational network

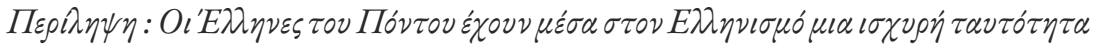

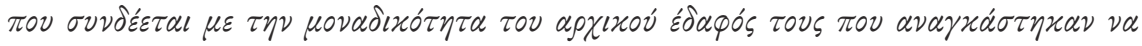

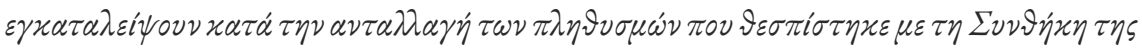

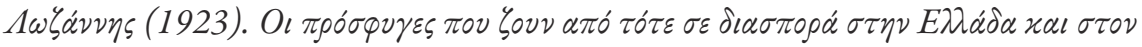

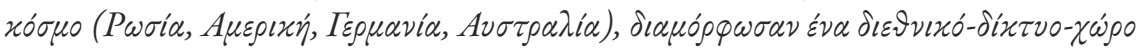

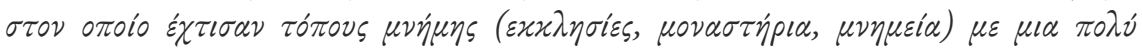

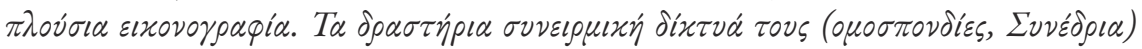

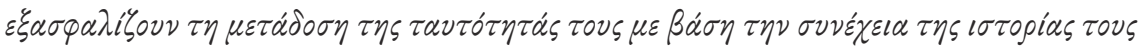

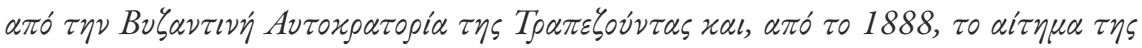

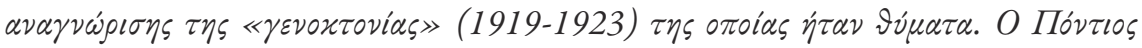

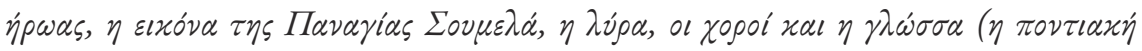

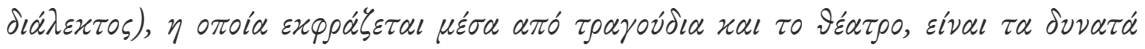

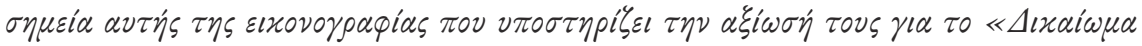

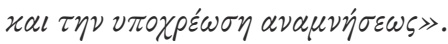

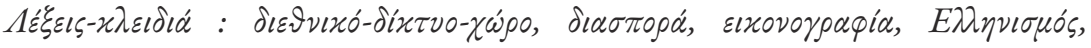

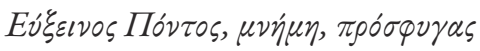

Anahtar Kelimeler: diaspora, Helenizm, ikonografi, bellek, Pondos, mülteci, ulusötesi ăg

Клучни зборови: дијаспора, хеленизам, иконографија, Понт Ексин, бегалии, транснационална мрежа 